\title{
Change detection of small objects and linear features in multi-temporal polarimetric images
}

Dierking, Wolfgang; Schou, Jesper; Skriver, Henning

Published in:

Proceedings of the International Geoscience and Remote

Sensing Symposium IGARSS 2000

Link to article, DOI:

10.1109/IGARSS.2000.857315

Publication date:

2000

Document Version

Publisher's PDF, also known as Version of record

Link back to DTU Orbit

Citation (APA):

Dierking, W., Schou, J., \& Skriver, H. (2000). Change detection of small objects and linear features in multitemporal polarimetric images. In Proceedings of the International Geoscience and Remote Sensing Symposium IGARSS 2000 (Vol. 4, pp. 1693-1695). IEEE. https://doi.org/10.1109/IGARSS.2000.857315

\section{General rights}

Copyright and moral rights for the publications made accessible in the public portal are retained by the authors and/or other copyright owners and it is a condition of accessing publications that users recognise and abide by the legal requirements associated with these rights.

- Users may download and print one copy of any publication from the public portal for the purpose of private study or research.

- You may not further distribute the material or use it for any profit-making activity or commercial gain

- You may freely distribute the URL identifying the publication in the public portal 


\title{
Change Detection of Small Objects And Linear Features in Multi-Temporal Polarimetric Images
}

\author{
Wolfgang Dierking, Jesper Schou, and Henning Skriver \\ Department of Electromagnetic Systems \\ Technical University of Denmark, Bldg. 348 \\ DK-2800 Lyngby, Denmark \\ Phone: +45 45253830, Fax: +45 45931634, Email: wd@emi.dtu.dk
}

\begin{abstract}
A SAR image sequence acquired over several months was used to study temporal variations of radar signatures of object classes which are relevant for updating topographic maps. For the selected object classes (buildings, roads, and hedgerows), the average pixel-to-pixel intensity variations are relatively stable over time intervals between data acquisitions from one month to one year. The threshold to be set in order to distinguish relevant changes from inherent signature variations decreases considerably, if signature mean values of individual objects are compared to each other rather than the signature values of individual pixels.
\end{abstract}

\section{INTRODUCTION}

At present, a research project "Topographic mapping by SAR" is carried out by the Danish Center for Remote Sensing, the Department of Planning (both at the Technical University of Denmark), and the Danish Survey and Cadastre (KMS). The project aims at exploring the potential of SAR to make the process of compiling and revising topographic maps more efficient (note that the term "topographic" here means thematic information of any kind, it is not restricted to elevation data). As one part of the project, it is investigated how well change detection of different object classes relevant for topographic mapping can be carried out utilizing multitemporal polarimetric SAR images.

The optimal prerequisite for change detection is that the images to be compared are measured with identical sensor parameters (same frequency, polarization, and illumination geometry). In practice, small differences between the flight tracks may cause slight changes of the illumination geometry. Several objects of interest reveal inherent temporal signature changes which are not subject of map updates. Intuitively, this is to be expected for many types of natural vegetation. As a first important step, we investigated the temporal signature stability of selected object classes. This gives a clue how large the effects of small deviations from an ideal flight track and of variations of dielectric and scattering properties on the observed signatures might be.

\section{DATA PREPARATION}

The image sequence used in this project was measured at $C$ and L-band by the Danish airborne EMISAR (a detailed description of the EMISAR is presented in [1]). The data were acquired over an agricultural test site in Jutland, Denmark, around the Research Center Foulum. The imaged area consist of smaller villages, agricultural fields, and small forests. Radar intensity images of the test area can be found, e.g., in [1] and [2]. The image sequence covers a time interval of five months from March to August 1998. At L-band, an additional image from June 1999 was used in our study. The nominal flight heading was identical for all flights. The local incidence angles at fixed ground range positions were slightly different due to variations in flight altitude. At C-band, the variations were from -1.3 to $+0.5 \mathrm{deg}$ at near range (reference angle $35 \mathrm{deg}$ ), and from -1.0 to +0.2 at far range $(60 \mathrm{deg})$. At L-band, the corresponding numbers are from -3.2 to $0.0 \mathrm{deg}$ at near range (reference angle $38.7 \mathrm{deg}$ ) and from -1.1 to 0.0 $\mathrm{deg}$ at far range $(61.2 \mathrm{deg})$. The reference angles given for L-band are for the same ground-range positions as the ones given for C-band.

For our study, we utilized the covariance matrix format which is an operationally provided EMISAR data product (see ref. [2] for details). In this format, the data are given in ground range projection with a pixel spacing of 5 by $5 \mathrm{~m}$. Neighboring pixels are correlated, the effective spatial resolution is about $8 \mathrm{~m}$. We chose the covariance matrix format because of its reduced speckle noise level (the effective number of looks for an individual pixel is $E N L=13$ ). From the covariance matrix elements, the backscattering coefficient $\sigma^{0}$ at VV-polarization is discussed in this paper. All images were registered to a digital elevation model (DEM) which was generated from interferometric airbome data acquired by EMISAR in 1997. Although the registration to a DEM was not necessary for a direct comparison of the different polarimetric images (since all data were acquired at an almost identical imaging geometry), it was nevertheless carried out in order to combine the radar images directly with aerial photographs, topographic maps, and vector layers representing different object classes. This complementary material was provided by KMS. The radar images were registered to one another with an rms-accuracy of better than one pixel. However, since we used nearest neighbor interpolation for the resampling of the images (in order not to reduce the effective spatial resolution), a deviation of one pixel was possible in small areas. This was taken into account in the further processing.

This work was supported by ESA Folgeforskningsfonden 


\section{SELECTION OF OBJECT CLASSES}

For our study, we focused on buildings, roads, and hedgerows which are examples of objects regularly monitored (by local authorities, e. g.). These objects are comparatively small. In most cases, buildings comprise only a small number of pixels in our radar images, and roads and hedgerows are only 1-3 pixels wide. Hence, they are often difficult to detect. The positions of the different objects in the radar images were identified by comparison with aerial photography. Straight line segments on roads and hedgerows as well as pixels covering buildings were marked. For crosschecking of radar signature characteristics, representatives of two additional object classes (forests and village background, the latter representing the area within a village not covered by buildings) were also marked and used in subsequent analysis.

\section{ANALYSIS OF SIGNATURE CHANGES}

Besides "relevant" changes of the radar signature at a given position, for example due to adding or removing an object, the signature might vary because of changes of scattering characteristics and dielectric properties, misregistration, and calibration errors. The latter two technical factors are of minor importance in the data set we used. The peak-to-peak difference of the backscattered intensity due to possible errors of the external calibration factors is smaller than $1.2 \mathrm{~dB}$ at $\mathrm{C}$-band and $2 \mathrm{~dB}$ at L-band. This was assessed on the basis of the relatively stable backscattering coefficients of forest areas (evaluated separately for each image, see Fig. 1). Temporal signature changes of different objects were studied by evaluating the pixel-to-pixel absolute difference between two images for different time intervals. The shortest time interval was three days between two data acquisitions in March 98, the longest interval we have included is one year (June 98 to June 99). The pixel-to-pixel difference of the backscattering coefficient $\sigma^{\circ}$ (in $\mathrm{dB}$ ), which for L-band is shown in Fig. 2, is on average more or less stable over time intervals between one month and one year (for a given time interval, variations of the average difference from image pair to image pair occur which depend on object type and radar band). In Fig. 2, all object classes except forest reveal a slightly smaller difference for the three days interval. As Fig. 1 indicates, the average difference for the three days interval may to a great extend be caused by a possible relative offset of the external calibration factors between the two March data acquisitions.

Changes of scattering characteristics at a given position cause variations of $\sigma^{0}$ and decrease the correlation of the speckle pattern between two images acquired over the same area. Because of the varying speckle patterns, the differences obtained for different pixels vary. Our results (including the analysis of $\sigma^{0}$ as a function of incidence angle for the different object classes similar to Fig. 1) indicate that the contribution of changes in the scattering characteristics is significant.

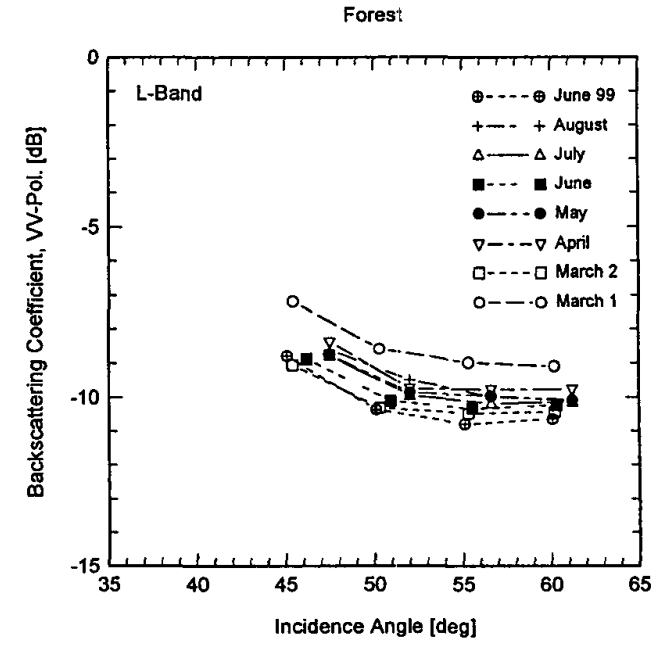

Fig. 1. Backscattering coefficients of forest at L-band, VVpolarization, evaluated from images acquired at different times. The number of averaged pixels was larger than 24000 , so that the effect of speckle can be neglected.

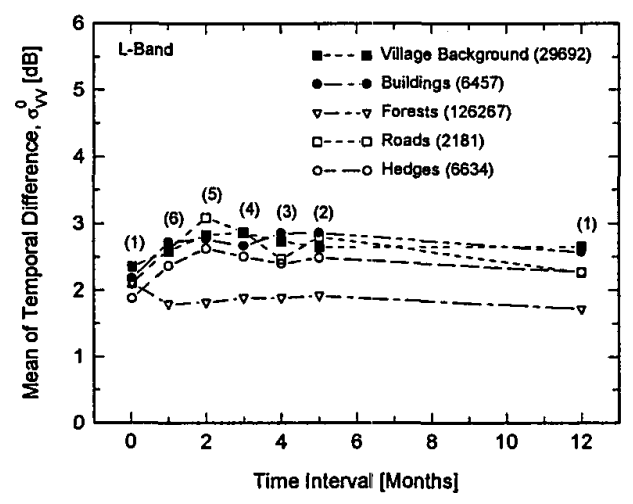

Fig. 2. Average absolute difference of the backscattering coefficient (in logarithmic scale) for different object classes as a function of the time interval between data acquisitions. Differences were evaluated pixel-to-pixel and then averaged over the whole incidence angle interval. The number of pixels for each object class is given in the legend. The numbers above the curves indicate possible combinations of images. For a 5-month interval, e. g., images from March, measurement 1 and 2, are combined with the image from August.

Often, changes between images are evaluated and interpreted on a pixel-to-pixel basis. In the case of radar images, this requires a considerable reduction of speckle noise, otherwise, the observed differences can be rather large (Fig. 3, lowermost graph). In order to preserve linear structures and small, point-like targets in the radar image, sophisticated filtering techniques are required [3]. Another possibility is to 
utilize masks which assign a group of pixels to a certain object. These masks can be generated, e. g., from vector layers showing the contours of different objects, as they are provided by KMS. The upper two histograms in Fig. 3 were generated by evaluating the temporal difference between the mean $\sigma^{0}$-values of each object obtained in the different images. It is obvious that this method allows a much lower threshold for change detection which is about $5 \mathrm{~dB}$ in the example shown in Fig. 3.

\section{DISCUSSION AND CONCLUSIONS}

The temporal signature variation inherent in radar images is considerable even if the differences in imaging geometry are very small. For hedgerows, this is due to the seasonal development of the leaves and small variations of the position of the individual leaves to which the radar is sensitive. Hedgerows are easy to detect in our radar images and usually wider than one pixel. Many roads in the imaged area, on the other hand, are difficult to identify. They are often less than one pixel wide and characterized by a low, partly highly variable backscattered intensity. The latter is due to the fact that the signature value which is assigned to a certain pixel might be a mixture of the radar response from the road's surface and from the adjacent vegetation (or adjacent buildings). Buildings (as stable objects) reveal relatively large temporal differences and difference variations. This needs to be investigated further.

We found that for the selected object classes, the inherent average pixel-to-pixel intensity variations between images acquired at different times (Fig. 2) increase within days and reveal only slight differences for time intervals between one and twelve months (less than $1 \mathrm{~dB}$ both at L- and C-band). Because of speckle decorrelation, average intensity values (in $\mathrm{dB}$ ) of individual objects should be used for change detection rather than pixel-to-pixel differences. A successful detection of "relevant" changes depends not only on the inherent signature variation of the different object classes but also on the signature contrast to the adjacent areas. The removal of a building, e. g., might not change the backscattered intensity to a level below or above the detection threshold, if the intensity level of the village background is very similar.

In this paper, we limited our examples to the L-band backscattering coefficient at VV-polarization. A complete report of our studies will be presented elsewhere.

\section{REFERENCES}

[1] E. L. Christensen et al., "EMISAR: An absolutely calibrated polarimetric L- and C-band SAR," IEEE Trans. Geosci. Remote Sensing, vol. 36, pp.1852-1865, Nov. 1998.

[2] H. Skriver, M. T. Svendsen, and A. G. Thomsen, "Multitemporal C- and L-Band Polarimetric Signatures of Crops," IEEE Trans. Geosci. Remote Sensing, vol. 37, pp.2413-2429, Sep. 1999.
[3] J. Schou, W. Dierking, and H. Skriver, "Multi-Look Polarimetric SAR Image Filtering Using Simulated Annealing," Proceedings of the IGARSS 2000, Hawaii, 2000.
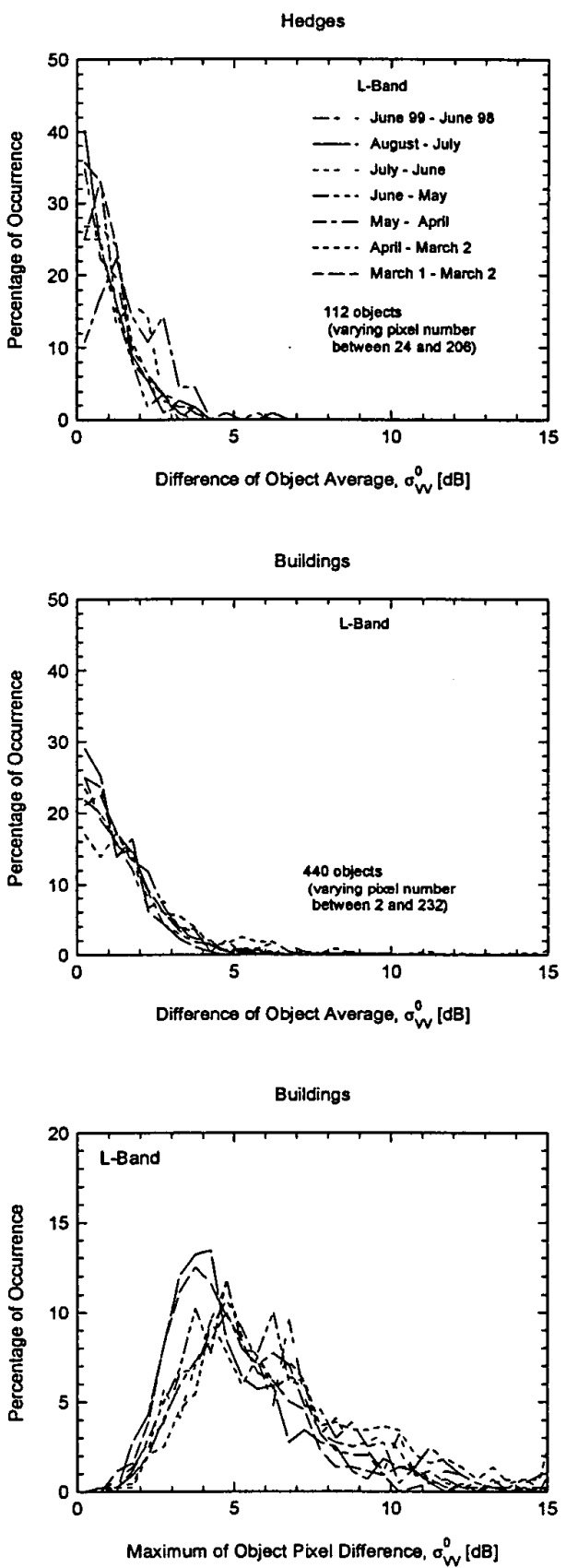

Fig. 3. Histogram of differences between object means for hedges and buildings (upper two graphs) and maximum pixel difference of each object for object class "buildings" (lowermost graph). 